\title{
MULTI-PARAMETRIC IRIS-PHOTOMETRY
}

\author{
T.B. GEORGIEV \\ Rozhen National Astronomical Observatory \\ P.O. Box 136 \\ $B G-4700$ Smolyan \\ Bulgaria
}

Usually, the reduction of iris-measurements $d$ of stellar photographic images to magnitudes $m$ is performed with one-dimensional regression $m=f(d)$, obtained using standard stars (Burkhead \& Seeds 1971). The derivation of accurate magnitudes is difficult due to many factors (Butler 1972). Considering that the star image shape contains information on the sensitivity of the plate and the color of the star, Vladimirov (1978) recommended multi-parametric determination of the magnitudes, using a system of isodense bounded areas of the stellar images. Here this approach is implemented using software that performs a general multi-parametric method (MPM) of irisphotometry.

MPM needs two or more systems of iris-measurements $d_{k}$ (corresponding to different comparative light beams), background measurements $d_{b}$ and, if the vignetting is strong, distances from the plate centre $r$. The initial magnitude estimations $m_{k}$ of the stars are determined from the multi-dimensional regression model $d_{\mathrm{k}}=f\left(m, d_{\mathrm{b}}, r^{2}\right)$, including up to third order term of $m$ and linear terms of $d_{b}$ and $r^{2}$. The final magnitude estimations $m$ are derived from the regression $m=g\left(m_{1}, m_{2}, \ldots\right)$.

The MPM were applied to the plates of the Bulgarian 2m RC telescope for the photometry of bright red stars in the nearby galaxy $M 33$. About 25 standard stars in the range 14 - 19 mag and three measurements modes were used. The typical standard deviation of the MPM photometry in this very difficult case is about $0.1 \mathrm{mag}$, whereas the classical one-dimensional approach gives $0.2-0.4$ mag. The color coefficient by the classical method, as well as by the first stage of the MPM, is about 0.1. It drops by a factor of 2 in the final of MPM, and the color reduction procedure (Garcia-Pelayo \& Alfaro 1982) is not necessary.

MPM is suitable for star photometry of large field plates with well-exposed backgrounds, however it needs rich standard sequences, well-distributed in magnitude, color, background and position.

\section{References}

Burkhead, M. and Seeds, M., 1971. AAS Photo Bull., 1, 15.

Butler, C.J., 1972. The Irish AJ., 10, 251.

Garcia-Pelayo, J.M. and Alfaro, E.J., 1982. Rev. Mexicana Astron. Astrophys., 5, 167.

Vladimirov, S.B., 1978. Astron. Tzirc., 980, 4. 